\title{
APA2I - Uma Arquitetura Pedagógica Aberta, Adaptativa e Inteligente para Construção Cooperativa de Conhecimento
}

\author{
Cristiano Biancardi - PPGI/UFES, cristiano.biancardi@gmail.com \\ Juliana Cristina dos Santos de Andrade - PPGI/UFES, \\ julianacristina.ti@gmail.com \\ Sidnei Santana - PPGI/UFES, sidneisc@gmail.com \\ Crediné Silva de Menezes - PPGIE/UFRGS, PPGI/UFES - credine@ gmail.com
}

Resumo. Este trabalho propõe uma Arquitetura Pedagógica para construção cooperativa do conhecimento, que fomenta a aprendizagem em rede, incentivando a interação e a crítica construtiva, e a mediação distribuída entre alunos e professor. Esta Arquitetura Pedagógica apresenta características inovadoras, por ser aberta, adaptativa e inteligente. Um experimento foi proposto e realizado a partir de um protótipo, que permitiu avaliar a efetividade da Arquitetura. Os resultados apresentaram-se promissores pelos bons níveis de aceitação dos atores envolvidos com relação aos indicadores avaliados, demonstrando a importância e relevância da abordagem proposta.

Palavras-chaves: arquitetura pedagógica, construção do conhecimento, cooperação, aprendizagem em rede, inteligência artificial.

\section{APA2I - An Open, Adaptive and Intelligent Pedagogical Architecture for Cooperative Knowledge Building}

\begin{abstract}
This work proposes a Pedagogical Architecture for cooperative knowledge building, which promotes networked learning, encouraging interaction and constructive criticism, and mediation distributed between students and professor. This Pedagogical Architecture presents innovative features, for being open, adaptive and intelligent. An experiment was proposed and performed by a prototype, which allowed to evaluate the effectiveness of the Architecture. The results were promising due to the involved actor's good levels of acceptance in relation to the evaluated indicators, showing the importance and relevance of the proposed approach.
\end{abstract}

Keywords: pedagogical architecture, knowledge building, cooperation, network learning, artificial intelligence.

\section{Introdução}

A Web 2.0 tem mudado a forma como as pessoas interagem. As suas ferramentas, segundo Rosen e Nelson (2008), apresentam características participativas, de compartilhamento de conteúdo e recursos, propiciando o desenvolvimento de comunidades sociais on-line. Embora não tenham sido projetadas para fins educacionais, Lee e McLoughlin (2007) ressaltam que as ferramentas da Web 2.0 oferecem oportunidades únicas, transformando o processo de ensino e a natureza das experiências de aprendizagem e têm um efeito positivo em vários desafios educacionais contemporâneos, como envolvimento do aluno, autonomia em aprender e a aprendizagem autêntica e significativa.

Porém, por mais sofisticada que seja determinada tecnologia, sua fronteira pedagógica encerra-se no limite da concepção de ensino e não propriamente de aprendizagem que alcança. Além disso, é necessário um aporte teórico para uso destas 
ferramentas didáticas, pois a falta dele resulta em uma utilização como "receita" ou "novidade", tornando-a descartável em um curto período de tempo (Carvalho et al, 2005).

Buscando contribuir com alternativas integradas, Carvalho et at (2005) apresentam as Arquiteturas Pedagógicas (APs). Para os quais, "as arquiteturas pedagógicas são, antes de tudo, estruturas de aprendizagem realizadas a partir da confluência de diferentes componentes: abordagem pedagógica, software, internet, inteligência artificial, educação a distância e concepção de tempo e espaço".

Neste ambiente ecossistêmico, formado pela combinação do aparato tecnológico com epistemologia e visão pedagógica, a tecnologia digital tem um papel fundamental pois, segundo Menezes et al. (2013) ela "não é entendida apenas como um suporte para as ações dos sujeitos, mas sim como elemento que também constitui as novas relações e formas de pensar.". Isso pressupõe uma sinergia do uso da tecnologia com as práticas pedagógicas, ressignificando os papéis e as formas de mediação, bem como oferece condições favoráveis para as construções coletivas.

Assim, neste contexto desafiador, o presente trabalho propõe uma nova Arquitetura Pedagógica, aberta, adaptativa e inteligente para construção do conhecimento em assuntos tratados em objetos de estudo a partir de produções individuais e coletivas, tornando possível o estabelecimento de uma rede de aprendizagem distribuída e cooperativa, incentivando a interação entre os sujeitos, a reflexão e a revisão por pares.

\section{Referencial Teórico}

O conceito de cooperação adotado em nossas pesquisas está apoiado na epistemologia genética. Piaget (1973, p. 22) define cooperação como "operações efetuadas em comum ou em correspondência recíproca". Em (Piaget, 1998), o autor ressalta que para que haja cooperação é necessário que os indivíduos possam pensar em torno de uma realidade comum, renunciando interesses próprios. Encontra-se ainda a ideia de que a coordenação dos pontos de vista e a descentração são condições necessárias para que a cooperação seja efetivada.

Scardamalia e Bereiter (2006) ressaltam a relevância do desenvolvimento do conhecimento em vez da transmissão dele. Os alunos precisam ser considerados como membros de uma comunidade de construção de conhecimento no lugar de simples aprendizes, possibilitando, assim, que a construção do saber aconteça através de um esforço coletivo e cooperativo, gerando o aprendizado individual.

Relacionado a isso, e pautado na ideia construtivista (Dias, 2010), em que as pessoas ativamente e progressivamente constroem ou fazem seu próprio conhecimento e que a realidade é determinada pelas experiências do aluno, as APs pressupõem a construção do conhecimento por meio da vivência de experiências e na ação dos sujeitos sobre o meio socioecológico, objetos e os fatos.

Para Nevado et al (2011), as APs propõem aos estudantes, atitudes ativas e reflexivas que, para a sua concretização, necessitam de suportes flexíveis e adaptáveis a diferentes contextos, capazes de gerar uma ampliação dos espaços e tempos da aprendizagem. Dessa forma, as APs pressupõem pesquisa, atividades interativas, autorais e co-autorais, envolvendo tecnologias e abordagens pedagógicas problematizadoras. Esses componentes atuam de forma a provocar, por um lado, desequilíbrios cognitivos e, por outro, suportes para as reconstruções. 
Seguindo uma perspectiva da ecologia cognitiva (Levy, 2010), as APs, conforme Nevado (2016a), são concebidas como "microecossistemas cognitivos" que englobam ideias epistemológicas relacionais, pedagogias abertas, tecnologias digitais e novos referenciais de tempo e espaço como condições "estruturantes" para as aprendizagens individuais e construções coletivas. A autora também reforça que nas APs as possibilidades interativas que as redes oferecem não podem ficar restritas à centralidade da figura do professor, sendo necessária a consolidação de uma política de envolvimento e um comprometimento dos sujeitos visando a construção coletiva, sendo necessária uma organização de trabalho que estabeleça a mediação não apenas como ação de um professor, mas sim como uma função distribuída entre os participantes.

\section{Trabalhos Relacionados}

Esta seção traz os principais trabalhos relacionados com a AP proposta, ou seja, aqueles que permitem uma melhor utilização de tecnologias digitais para o ensino, aliando teorias pedagógicas.

A proposta da AP Elaboração de Histórias Coletivas (Nevado et al, 2016b) é fomentar a criatividade e a articulação textual dos participantes em conseguir elaborar, de forma cooperativa e à distância, sequências contextualizadas para uma história. Os grupos escolhem o gênero literário da história, definem um título e escrevem o primeiro parágrafo. A partir disso, os alunos constroem o restante da história, onde cada membro do grupo necessita escrever, de forma intercalada, um parágrafo articulado e coerente com os parágrafos anteriores, com o gênero e com o título. A quantidade de rodadas para o desenvolvimento do texto é combinada a priori. Ao final, cada grupo realiza uma discussão para avaliar: a) a qualidade do texto produzido, em particular, a coerência entre os parágrafos e, b) o desenvolvimento individual com respeito as competências para cooperar . Para suportar esta AP foi desenvolvido o ambiente EsCola (Barros e Menezes, 2016).

Na AP Debate de Teses (Nevado, 2011), os alunos desenvolvem um debate a partir de teses iniciais. Cada participante deve se manifestar a respeito de cada tese, indicando se concorda, discorda ou não sabe opinar, argumentando sua justificativa. Cada argumentação deve ser lida por, pelo menos outros dois alunos, a fim de validar as justificativas do colega. $\mathrm{O}$ autor do argumento, por sua vez, deve tomar conhecimento das revisões e, com base nelas, elaborar uma réplica, apresentado explicações, contestações e convergências com o posicionamento dos revisores. Ao mesmo tempo que um sujeito tem suas argumentações revisadas, ele também revisa as argumentações de outros colegas. Ao final, espera-se que os participantes possam reelaborar seu posicionamento inicial sobre as teses discutidas. Para esta AP foi desenvolvido um ambiente para a realização das tarefas propostas (Nevado, 2011) e (Fernandes-Jr; Menezes, 2015).

Na AP Socializando Soluções (Marques e Tavares, 2015), o professor solicita que os alunos resolvam uma lista de exercícios de programação de computadores e socializem suas soluções com os demais alunos, de forma que eles poderão acessar, analisar, testar e comentar as soluções dos colegas. O professor decide se aqueles que não conseguiram resolver a lista podem ter acesso às soluções do demais colegas, para apoiar a elaboração de suas próprias respostas. Para esta AP foi desenvolvido um ambiente para atender às suas necessidades.

A AP Auxílio na Educação de Surdos (Santos, Ricardo et al, 2007) é destinada à formação de professores que lidam com alunos surdos. Sua dimensão pedagógica se pauta no construtivismo social, no respeito e na identidade linguística dos aprendizes. 
Como suporte computacional à AP, foi utilizado o ambiente PII (Plataforma Interativa para Internet) e um sistema de autoria de material didático para surdos.

A AP apresentada neste artigo está em sintonia com os trabalhos relacionados, fornecendo apoio pedagógico para a inserção de tecnologias digitais no processo de ensino, ampliando os espaços para a interação e cooperação entre os participantes e buscando potencializar as aprendizagem dos alunos. Adicionalmente, contribui com uma nova concepção pedagógica e traz elementos inovadores em seu suporte computacional relacionados à aplicação de inteligência artificial para promover, por exemplo, adaptatividade, análise de aprendizado e interação, customização e recomendação.

\section{Arquitetura Pedagógica Proposta}

A proposta deste trabalho se baseia em Carvalho et al (2005), que estabelece o conceito de AP sustentada em três componentes: 1) Concepção pedagógica; 2) Sistematização metodológica buscando proporcionar aos estudantes, atividades intelectuais interessantes; e 3) Suporte computacional de apoio ao processo.

\subsection{Concepção Pedagógica}

A concepção desta AP, baseia-se na construção do conhecimento dos alunos em assuntos tratados em Objetos de Estudo (OEs) (artigos científicos, reportagens, vídeos, visita técnica/educacional, peça de teatro, etc), a partir da elaboração de produções (relatórios, resenhas, fichamentos, etc) individuais e em grupo; tendo a cooperação (Piaget, 1973, p. 22), interação em rede, a reflexão e a revisão por pares como pilares.

\subsection{Sistematização Metodológica}

Visando a efetiva participação e contribuição de cada sujeito para o processo, foram definidos os seguintes passos estruturantes para a sistematização (Figura 1):

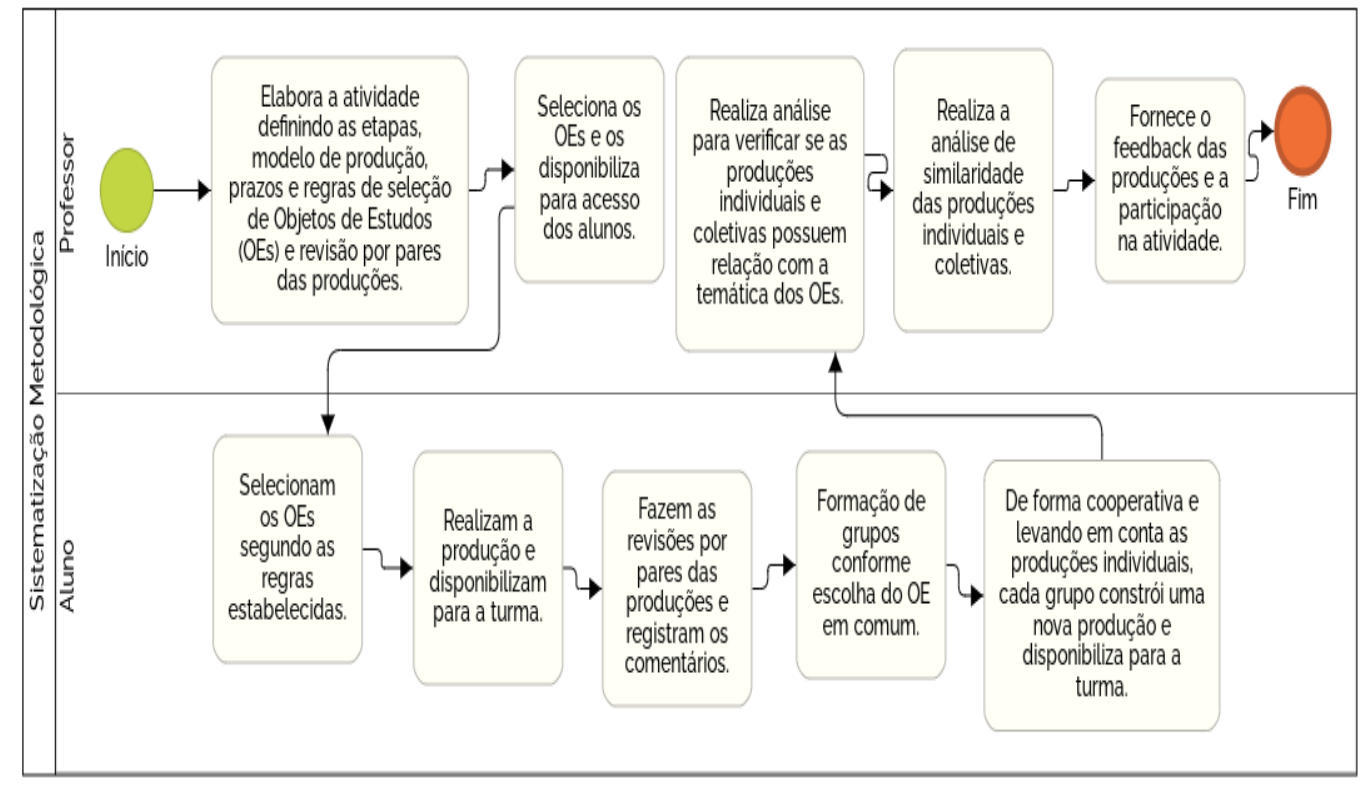

Figura 1 - Passos estruturantes da sistematização metodológica da AP proposta.

\subsection{Suporte Computacional}

Para as características específicas desta AP e levando em conta os resultados do experimento desenvolvido (Seção 5), os recursos computacionais (Figura 02) foram 
definidos através de um ambiente multiplataforma, usando arquitetura cliente-servidor baseada em componentes visando o reuso e integração.

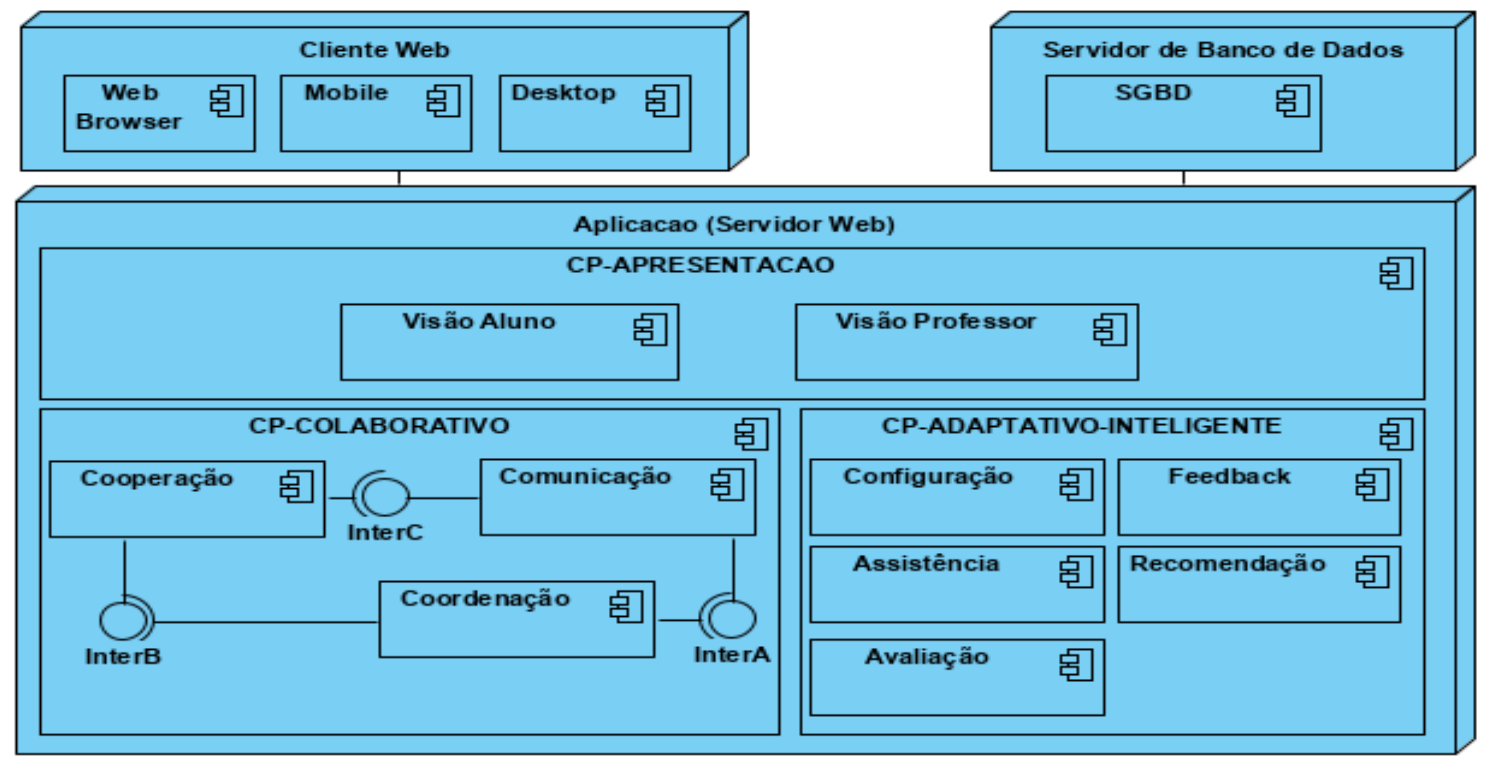

Figura 2 - Diagrama de arquitetura do ambiente computacional proposto.

\subsubsection{CP-APRESENTAÇÃO}

Para controlar o acesso dos usuários e suas visões ao sistema, o componente CPAPRESENTACAO, segundo o tipo de usuário e níveis de permissão, ativará as funcionalidades dos demais componentes na camada de Aplicação e personalização das interfaces para os atores envolvidos.

\subsubsection{CP-COLABORATIVO}

No suporte computacional às dinâmicas cooperativas de nossa proposta, levaremos em conta os elementos do Modelo 3C proposto por Ellis et al (1991). Este modelo tem sido usado na literatura para classificação dos sistemas colaborativos e se baseia em três componentes inter-relacionados: Comunicação (troca de informações), Coordenação (atividades a serem realizadas) e Cooperação (operação conjunta dos membros do grupo em um espaço compartilhado). O CP-COLABORATIVO oferece suporte a cada um dos componentes do Modelo 3C:

i) Cooperação: funcionalidades para suportar a produção conjunta dos membros do grupo em um espaço compartilhado, gerando e manipulando objetos de cooperação na realização das tarefas; ii) Comunicação: funcionalidades de fórum e chat disponíveis, permitindo interações e discussões sobre as atividades a serem desenvolvidas. Além disso, o ambiente também suporta envio de alertas sobre prazos e pendências em atividades, baixo índice de participação dos alunos e mensagens motivacionais; iii) Coordenação: funcionalidades de planejamento da atividade definindo suas etapas, workflow entre elas, criação/customização/seleção de modelos de produções, definição dos prazos, seleção e disponibilização de OEs para a atividade, regra de revisão por pares, regra de escolha de OEs pelos alunos, formação (semi) automática de grupos, controle de acesso para as produções dos alunos e configurações diversas.

\subsubsection{CP-ADAPTATIVO-INTELIGENTE}


No contexto de utilização com grandes contingentes de alunos, faz-se necessário prover facilidades para que os professores possam desempenhar seu trabalho com mais facilidade. Além disso, com a utilização frequente dessa AP, um volume considerável de dados será gerado, possibilitando que diferentes análises possam ser realizadas e os resultados usados para potencializar o trabalho dos professores e o aprendizado dos alunos. Para este contexto, os seguintes componentes estão previstos:

i) CONFIGURAÇÃO: a partir da análise de dados de perfis, formas de interação e dos resultados alcançados pelos alunos no desenvolvimento das atividades propostas, torna-se possível fazer indicações do que funcionou bem para tipos específicos de grupos de alunos, podendo o professor, a partir de indicações automáticas feitas pela AP, selecionar tipos específicos de OEs, cenários de atividades para alunos com estilo e padrão de aprendizagem semelhantes e/ou distintos;

ii) ASSISTÊNCIA: a coleta e análise de dados em espaços de interação, por exemplo em fórum de discussão, pode encontrar padrões de respostas para dúvidas e discussões comuns e, dessa forma, gerar insumos para alimentar continuamente um chatbot (Kuyven et al, 2018) que tire dúvidas. Essa possibilidade pode ser muito útil para encontrar respostas rápidas, antes inclusive de acessar o fórum ou a página de perguntas e respostas, podendo evoluir para um assistente pessoal de aprendizado acoplado à AP auxiliando o professor no assessoramento dos alunos;

iii) FEEDBACK: avaliação de desempenho dos alunos, comparando os resultados individuais e coletivos, possibilitando ao professor formular novas estratégias para ensinar os pontos em que um ou mais alunos estejam encontrando dificuldades de assimilação. Isso também é relevante para avaliar a efetividade de tipos distintos de configuração da AP e, com isso, saber o que pode ser modificado e propor novas formas de composição e descartar aquelas em que os alunos não apresentaram bom desempenho;

iv) RECOMENDAÇÃO: para a criação de novas atividades, o ambiente irá dispor da recomendação para o professor de novos OEs baseado nos OEs e assuntos tratados em atividades anteriores, na percepção de qualidade e desempenho dos alunos relacionado aos OEs. Para os alunos que desejam aprofundar conhecimento ou que estejam com dificuldade de entendimento dos assuntos abordados, o ambiente terá a opção de oferecer novos OEs para cada situação. Deste modo, um sistema de recomendação tem um papel importante para ajudar professores e alunos a encontrarem recursos educacionais relevantes e pertinentes aos seus perfis e aos contextos trabalhados (Laisa et al, 2018);

v) AVALIAÇÃO: com relação às produções dos alunos, individuais e cooperativas, serão realizadas análises a partir de técnicas de inteligência artificial (Baeza-Yates; Ribeiro-Neto, 2011) para averiguar o percentual de autoria individual para a versão cooperativa da produção, se possuem relação com a temática dos OEs e agrupamentos de produções similares e diferentes. Isso possibilitará ao professor gerar feedbacks de forma mais facilitada, levando em conta grupos de soluções e direcionar instruções para aquelas produções que estejam fugindo do escopo dos OEs considerados para o desenvolvimento da atividade.

\section{Experimentando a Arquitetura Proposta}

Para validação da efetividade da AP proposta, um experimento foi realizado com o objetivo principal de compreender a evolução do aprendizado, os desafios e problemas enfrentados por alunos e professor para a realização de uma atividade cooperativa nos moldes proposto pela AP em questão. Para viabilizar o experimento, foi criado um 
protótipo a partir de uma combinação de facilidades fornecidas por diferentes ferramentas (pbworks, google docs, hangouts, e-mail e whatsapp) que permitiram operacionalizar a execução da sistematização metodológica da AP. O experimento ocorreu na disciplina "Inteligência Artificial Aplicada à Educação", ofertada na modalidade a distância para alunos de pós-graduação de mestrado e doutorado em computação de uma instituição de ensino. O experimento foi conduzido da seguinte forma:

i) Em um primeiro momento o professor estabeleceu as etapas da atividade, selecionou e disponibilizou os OEs e definiu a estrutura da produção. Optou-se por artigos científicos e a produção escolhida foi o fichamento. Dos artigos disponibilizados, dois deles deveriam ser lidos e fichados por todos os alunos. Um terceiro deveria ser escolhido pelos alunos para leitura e fichamento, de forma que um mesmo artigo tivesse apenas dois alunos associados.

ii) Após a etapa de fichamentos individuais, os alunos disponibilizaram as produções e o professor organizou os grupos de revisão por pares, de forma que cada fichamento fosse revisado por dois alunos. Nesta etapa, cada aluno lançou comentários sobre os fichamentos de seus pares e os proprietários das produções individuais interagiram respondendo às críticas feitas.

iii) Na parte final, os alunos se dividiram em grupos, levando em conta a escolha comum do artigo, para discutir o tema e produzir, de forma cooperativa, um novo fichamento. Ao final, o professor analisou cada produção e realizou a interação coletiva com os alunos para tirar conclusões, lançar novas questões e fornecer o feedback sobre os resultados, gerando conhecimento e evolução no aprendizado.

\section{Resultados e Discussões}

O experimento foi conduzido em julho de 2020. Na disciplina estavam matriculados 11 alunos. Ao final da atividade, foi realizada uma pesquisa com os alunos e professor visando captar a percepção sobre a AP, em diferentes aspectos. A partir das respostas de 09 alunos e do professor, foi possível realizar algumas análises, apresentadas seguir.

\subsection{Percepção dos Alunos}

Os alunos responderam a um questionário com 08 perguntas fechadas e 03 abertas. Objetivou-se medir aspectos relacionados a: i) construção do conhecimento, gerando o aprendizado individual; ii) clareza e reflexão na elaboração dos textos para facilitar o entendimento; iii) reconstrução dos conhecimentos prévios a partir de críticas; iv) promoção de atitudes proveitosas e reflexivas no âmbito individual e coletivo; v) promoção da mediação distribuída entre alunos e professor; vi) busca por subsídios complementares (pesquisas e leituras) para a construção das produções; vii) participação dos alunos de forma ativa em nível colaborativo; viii) recomendação da atividade; ix) aspectos relacionados ao suporte computacional utilizado.

Para responder aos indicadores de i) a vii), os alunos usaram uma escala likert de cinco pontos (1 a 5) para indicar o nível de percepção. Para uma melhor análise dos resultados, foi realizada uma abordagem quantitativa para estabelecer o Ranking Médio (RM) para mensurar o grau de concordância dos sujeitos que responderam o questionário. Conforme observado na Tabela 1, a percepção dos alunos é bastante satisfatória, com todos os resultados apresentando valores de RM maior ou igual a 4. Quando questionados se recomendariam esta atividade para algum professor aplicar no contexto de suas disciplinas, o indicador Net Promoter Score (NPS) apresenta um resultado de 77.8, indicando nível de excelência. 
Tabela 1 - Ranking Médio dos indicadores i) a vii) - percepção dos alunos.

\begin{tabular}{|l|c|}
\hline \multicolumn{1}{|c|}{ Indicador } & RM \\
\hline i) construção do conhecimento, gerando o aprendizado individual. & 4.6 \\
\hline ii) clareza e reflexão na elaboração dos textos para facilitar o entendimento. & 4.0 \\
\hline iii) reconstrução dos conhecimentos prévios a partir de críticas. & 4.3 \\
\hline iv) promoção de atitudes proveitosas e reflexivas no âmbito individual e coletivo. & 4.5 \\
\hline v) promoção da mediação distribuída entre alunos e professor. & 4.3 \\
\hline vi) busca por subsídios complementares (pesquisas e leituras). & 4.8 \\
\hline vii) participação dos alunos de forma ativa em nível colaborativo. & 4.2 \\
\hline
\end{tabular}

Com relação às percepções originadas a partir do suporte computacional utilizado no experimento, considerando o trabalho dos participantes, os estudantes relataram: a) aspectos negativos - inexistência de uma plataforma única que suportasse o trabalho como um todo; falta de notificações para saber quando um revisor deixou um comentário na plataforma; falta de envio de alertas sobre prazos, lembretes para atuar na atividade devido a relação de dependência com a atividade posterior, falta de segurança devido a possíveis alterações nas produções dos pares, inteligência para organização das alocações para o professor (revisão por pares, artigos por pessoa); b) aspectos positivos - as ferramentas utilizadas permitiram realizar o trabalho colaborativo e cooperativo entre os participantes, coautoria, diálogo, flexibilidade para criação de páginas, inserção de comentários nas páginas para revisão por pares, visualização das produções dos colegas, cooperação simultânea na construção dos textos, possibilidade de comunicação a distância, geração de histórico, possibilidade de interação assíncrona e realizada a distância, persistência das produções para posterior consulta.

Os estudantes também foram convidados a fazer comentários gerais sobre a atividade. A partir deles foi possível constatar que a atividade favoreceu o trabalho em equipe de forma efetiva, a análise de diferentes pontos de vista sobre os objetos de estudo, a troca de experiência cultural de pessoas de diferentes lugares e a criatividade. Houve relato sobre a falta de mais co-mediação por parte dos alunos e cumprimento de prazos das atividades, pois muitas atividades impactam o trabalho coletivo. Também indicaram situações com possibilidades de automatização, por exemplo: avaliação da relação das contribuições dos alunos para o tema discutido; identificação de ideias semelhantes e divergentes; identificação do nível de participação dos alunos e suas dificuldades (exposição de ideias, diálogo, aceitação de críticas, etc.).

\subsection{Percepção do Professor}

A partir das respostas do professor pode-se fazer algumas considerações relacionadas ao processo cognitivo e às práticas educativas: a) houve compartilhamento de conhecimentos adquiridos pelos alunos proporcionado pela interação com as construções individuais e coletivas; b) através da revisão por pares e das interações para a produção cooperativa, ocorreram desequilíbrios cognitivos que impactaram positivamente os alunos e os levaram a repensar suas produções, criando novos possíveis; c) existe a necessidade de entendimento do verdadeiro propósito da crítica e de tomar consciência de que ela proporciona grandes possibilidades de evoluir o aprendizado, pois permite estabelecer reflexões, esclarecimentos de dúvidas e enxergar 
novos caminhos para a reconstrução dos saberes, e que por isso os alunos devem estar preparados para fazer e receber críticas; d) as práticas tradicionais de ensino são as mais aplicadas na formação dos alunos e a cultura do trabalho cooperativo é algo em desenvolvimento, assim, a cooperação através de um trabalho composto por etapas visando a criação de um produto final a ser alcançado pela contribuição coletiva, é uma estratégia que os alunos precisam experimentar com mais frequência para melhor aproveitamento.

\section{Considerações Finais}

As APs encerram uma interdependência de concepções pedagógicas, metodologias interativas e tecnologias digitais, propiciando a transformação do ambiente de ensino e aprendizagem, valendo-se da criatividade, reflexão, contestação e inovação. Esse enriquecimento de ideias torna o processo não apenas mais didático e útil ao aluno, como mais eficiente, propiciando diferentes estímulos e processos onde não apenas alunos e professores podem se beneficiar, mas toda a sociedade, com a geração de ideias e projetos inovadores, que podem impactar positivamente no dia-a-dia das pessoas.

Neste artigo propomos uma AP aberta, adaptativa e inteligente para construção de conhecimento de forma colaborativa, que favorece a interação em rede, cooperação, reflexão e revisão pelos pares. Apresentamos uma experiência de uso desta AP, por meio de um protótipo utilizando a ferramenta PBWorks e outras ferramentas externas. Este caso de uso teve como objetivo validar a efetividade da AP proposta, permitindo compreender a evolução do aprendizado, a construção do conhecimento, e os desafios e problemas enfrentados por alunos e professores.

Os resultados da experiência analisada mostraram que a AP alcançou seus objetivos, mostrando percepções positivas dos alunos relacionadas à construção de novos conhecimentos e reconstrução de outros prévios, produção de aprendizado individual, desenvolvimento de atitudes reflexivas no âmbito individual e coletivo, mediação distribuída entre alunos e professor e participação ativa dos alunos em nível colaborativo e cooperativo. Com relação à percepção do professor, a AP proporcionou um cenário para a prática da responsabilidade compartilhada, e isso impactou no desenvolvimento inicial de atitudes proativas dos alunos, desempenhando tarefas que ajudaram no bom andamento da atividade e no desenvolvimento da aprendizagem coletiva.

Por fim, para que todos os envolvidos tenham condições mais favoráveis de desenvolver suas atividades e aproveitarem do potencial que a AP pode oferecer, tornase necessário em trabalhos futuros, a implementação da solução computacional proposta.

\section{Referências Bibliográficas}

BAEZA-YATES, R.; RIBEIRO-NETO, B. Modern Information Retrieval: The Concepts and Technology Behind Search. UK: AddisonWesley, 2011.

BARROS, E. C.; MENEZES C. S. EsCola - Ambiente Educacional para Escrita Colaborativa. In SIMPÓSIO BRASILEIRO DE INFORMÁTICA NA EDUCAÇÃO, v.1, p. 1255, 2016.

CARVALHO, M. J. S.; NEVADO, R. A.; MENEZES, C. S. Arquiteturas pedagógicas para Educação a Distância: concepções e suporte telemático. In: SIMPÓSIO BRASILEIRO DE INFORMÁTICA NA EDUCAÇÃO, v.1, p.362-372. 2005. 
DIAS, I. S. Competências em educação: conceito e significado pedagógico. Psicologia Escolar e Educacional. Campinas, v. 14, n. 1, p. 73-78, 2010.

ELLIS, C. A.; GIBBS, S. J.; REIN, G. L. Groupware: some issues and experiences. Communications of the ACM, v. 34, n. 1, p. 39-58, 1991.

FERNANDES-Jr, J.I.C.; MENEZES, C.S. Apoio à Coordenação do Debate de Teses In: VII SEMANA DE INFORMÁTICA CESIT/UEA, v. 3, n. 1, 2015.

KUYVEN, N. L.; ANTUNES, C. A.; VANZIN, J. B.; SILVA, J. L. T.; KRASSMANN A. L.; TAROUCO, L. M. R. Chatbots na educação: uma Revisão Sistemática da Literatura. RENOTE - Revista Novas Tecnologias na Educação. v. 16, n. 2, 2018.

LAISA, J.; MEDEIROS, T.; ARANHA, E.; SILVA, T. R. Uma Revisão Sistemática da Literatura sobre Sistemas de Recomendação Educacional. In: COMPUTER ON THE BEACH, 2018, Florianópolis, SC. p.751-760.

LEE, M. J. W.; MCLOUGHLIN, C. Teaching and learning in the Web 2.0 era: empowering students through learner-generated content. International Journal of Instructional Technology and Distance Learning, 4(10), 1-17, 2007.

LÉVY, P. As tecnologias da inteligência: o futuro do pensamento na era da informática. São Paulo: Editora 34, 2010.

MARQUES, G. R.; TAVARES, O. L. Arquitetura pedagógica para aprendizagem de programação. In NUEVAS IDEAS EN INFORMATICA EDUCATIVA. Santiago. 2015.

MENEZES, C. S.; NEVADO, R. A.; ZIEDE, M. L.; CHARCZUK, S. B. Arquiteturas Pedagógicas para a Aprendizagem em Rede no Contexto do Seminário Integrador. RENOTE - Revista Novas Tecnologias na Educação, vol. 11, n. 02, 2013.

NEVADO, R. A.; MENEZES, C. S.; JUNIOR, R. R. V. (2011). Debate de Teses - Uma arquitetura pedagógica. In SIMPÓSIO BRASILEIRO DE INFORMÁTICA NA EDUCAÇÃ̃, v.1, 2011.

NEVADO, R. A. Interação e mediação no contexto das arquiteturas pedagógicas para a aprendizagem em rede. Revista de Educação Pública, v. 25, n. 59/1, p. 261-275, $2016 \mathrm{a}$.

NEVADO, R. A.; CHARCZUK, S. B.; ZIEDE, M. Uma arquitetura pedagógica na elaboração de histórias coletivas. In CONGRESSO BRASILEIRO INFORMÁTICA NA EDUCAÇÃO, v.5, p. 569, 2016 b.

PIAGET, J. Estudos sociológicos. Rio de Janeiro: Forense. 1973.

PIAGET, J. Sobre a Pedagogia: textos inéditos. São Paulo: Casa do Psicólogo, 1998.

ROSEN, D.; NELSON, C. Web 2.0: A New Generation of Learners and Education. Computers in the Schools, v. 25, p. 211-225, 2008.

SANTOS, Leonardo.; CASTRO, A.; MENEZES, C. Flexible virtual environments for teaching and learning. In: FRONTIERS IN EDUCATION CONFERENCE. 2012.

SANTOS, Ricardo. M.; ELIA, M. F.; SANTOS, M. P.; MORENO, M. P. R. Proposta de arquitetura pedagógica para auxiliar formadores na educação de surdos. In SIMPÓSIO BRASILEIRO DE INFORMÁTICA NA EDUCAÇÃO, v.1, p. 145154, 2007.

SCARDAMALIA, M.; BEREITER, C. Knowledge building: Theory, pedagogy, and technology. In K. Sawyer (Ed.), Cambridge Handbook of the Learning Sciences. New York: Cambridge University Press, p. 97-118, 2006. 